\title{
Connaissances, attitudes et pratiques des jeunes lycéens de Niono sur les IST, le VIH et le SIDA
}

\section{Knowledge, attitudes and practices of young high school students in Niono on STI / HIV / AIDS}

Sangho $\quad \mathbf{O}^{1,2,3^{*}}$,Telly $\quad \mathrm{N}^{2}$,Sogodogo $\quad \mathrm{A}^{4}$, Diarra $\mathrm{MM}^{5}$,Touré $\quad \mathrm{AY}^{4}$,Sangho $\quad \mathrm{F}^{1,2,6}$,Sangho $\quad \mathrm{A}^{1,6}$, Coulibaly $\mathrm{CA}^{2}$, Tounkara $\mathrm{M}^{2}$,Berthé $\mathrm{M}^{7}$, Kéita $\mathrm{I}^{3}$, Diop $\mathrm{S}^{2}$,Sangho $\mathrm{H}^{2}$

1 : Faculté de Pharmacie /USTTB, Mali

2 : Département d'Enseignement et de Recherche en Santé Publique, FMOS/USTTB, Mali

3 : Agence Nationale de Télésanté et d'Informatique Médicale, Bamako, Mali

4 : Centre de Santé de Référence de Niono, Ségou, Mali

5 : Centre de Santé de Référence de Kéniéba, Kayes, Mali

6 : Direction de la Pharmacie et du Médicament, DPM, Bamako, Mali

7 : Institut National de la Santé Publique

*Auteur correspondant : Dr Oumar Sangho, Email : osangh2005@gmail.com

\section{Résumé :}

Objectif : L'objectif était d'étudier les connaissances, attitudes et pratiques des jeunes lycéens de Niono en matière d'IST, de VIH et du SIDA.

Matériel et méthodes : Il s'agissait d'une étude transversale ayant concerné 369 élèves du lycée MICHEL ALLAIRE et du lycée Public L'étude s'est déroulée d'avril 2016 à mars 2017. L'échantillon a été aléatoire et l'interview en mode face à face. Les données ont été analysées sur SPSS 20.0.

Résultats : L’âge moyen était de 17,62 ans $\pm 1,8$ ans. Le sexe masculin prédominait avec $56 \%$. La majorité avait entendu parler des IST, du VIH et du Sida (95,90\%), beaucoup plus lors des causeries (43,9\%). Environ $42 \%$ et $46 \%$ ne connaissaient pas de signes d'IST et ne savaient qu'est-ce que c'est qu'une séropositivité. Les rapports sexuels étaient cités par $32 \%$ élèves comme voies de transmission. La majorité (74\%) savait que le multiple partenariat augmentait le risque de ces maladies. Le préservatif était le moyen de prévention le plus cité soit $44,2 \%$. Près de $63 \%$ ont accepté volontairementle test de dépistage. Un quart avait affirmé avoir réalisé le test et $35,2 \%$ avait déjà eu un contact sexuel dont 18,5\% était âgé de 12 à 14 ans. La curiosité fut la raison évoquée par $68,5 \%$. Parmi ceux en activité sexuelle, 45,4\% n'ont pas utilisé le préservatif au cours du premier rapport sexuel.

Conclusion :Cette étude nous montre une bonne connaissance des élèves sur l'existence des IST, VIH et sida, malgré quelques disparités avec leursattitudes et pratiques sexuelles.

Mots clés : attitudes, connaissances, IST/VIH/SIDA, lycéens, Niono, pratiques

Abstract

Objective: The objective was to study the knowledge, attitudes and practices of young high school students in Niono regarding STI and HIV-AIDS.

Material and methods: This was a cross-sectional study involving 369 students from the MICHEL ALLAIRE high school and the Public high school. The study took place from April 2016 to March 2017. The sample was random and the interview in face-to-face mode. The data was analyzed on SPSS 20.0.

Results: The mean age was 17.62 years \pm 1.8 years. Male was more frequent with $56 \%$. The majority had heard of STIs, HIV and AIDS (95.90\%), much more during chats (43.9\%). About $42 \%$ and $46 \%$ knew no signs of STIs or HIV infection. Sex was cited by $32 \%$ as a route of transmission. The majority $(74 \%) \mathrm{knew}$ that multiple partnerships increased the risk of these diseases. The condom was the most cited means of prevention with 44.2\%. Almost $63 \%$ said that they accepted voluntary testing. A quarter said they had taken the test and $35.2 \%$ already had sexual contact, including $18.5 \%$ between 12 and 14 years old. Curiosity was the reason cited by $68.5 \%$. Among those in sexual activity, $45.4 \%$ did not use a condom during the first sexual intercourse.

Conclusion: This study shows a good knowledge of the students about the existence of STIs, HIV and AIDS, despite some disparities with their sexual attitudes and practices.

Keywords: attitudes, knowledge, STI / HIV / AIDS, high school students, Niono, practices. 


\section{INTRODUCTION}

Selon le rapport de l'Organisation des Nations Unies pour la lutte contre le Syndrome d'Immunodéficience Acquisse (SIDA ONU) 2019 on estimait à l'échelle mondiale, 37,9 millions $(32,7-44,0)$ de personnes vivant avec le Virus de l'Immunodéficience Humaine $(\mathrm{Pv} \mathrm{VIH})$ dont 770 000 [570 000 - 1,1 million] décès [1]. En 2008 en Afrique sub-saharienne, on comptait plus de 25 millions de séropositifs et elle héberge environ $70 \%$ des personnes infectées de la planète [2]. La pauvreté, l'inégalité entre les sexes et le déplacement des personnes du fait d'un conflit ou d'une catastrophe naturelle constituent les facteurs socioéconomiques susceptibles d'accroître la vulnérabilité des personnes à l'infection au VIH [2]. La pandémie du VIH/Sida affecte principalement les populations jeunes en Afrique avec une prédominance de la transmission sexuelle [3]. En Afrique subsaharienne, le pourcentage de jeunes (15-24 ans) possédant des connaissances complètes et pertinentes sur le VIH a augmenté de 5 points chez les hommes et de 3 chez les femmes entre 2002 et 2011 [2]. Cependant, le niveau de connaissance dans ce domaine reste faible $(36 \%$ chez les jeunes hommes et $28 \%$ chez les jeunes femmes)[1]. Selon l'enquête démographique et santé du Mali (EDSM-V 2012), réalisée sur l'ensemble du pays excepté les régions de Gao, Tombouctou et Kidal, la prévalence de l'infection par le VIH au sein de la population générale au Mali est estimée à $1,1 \%$ et parmi les jeunes de 15 24 ans elle est de $0,8 \%$ et à cette période de la vie, les relations sexuelles sont généralement instables et la pratique du multi partenariat sexuel plus fréquente, ce qui augmente leur risque [4]. Parmi les jeunes femmes de 15-24 ans, $24 \%$ sont considérées comme ayant une connaissance jugée comme « approfondie » du sida. Cette proportion est inférieure à celle des hommes (33\%). On constate que les rapports sexuels à hauts risques (rapports sexuels avec un partenaire extraconjugal et non cohabitant) sont plus fréquents parmi les jeunes de niveau secondaire et plus avec $47 \%$ contre seulement $8 \%$ parmi celles qui n'ont aucun niveau ; et l'utilisation d'un préservatif lors des rapports sexuels à hauts risques chez ces jeunes de 15-24 ans apparaît faible, seulement (18\%) ont déclaré avoir en utilisé au cours de leurs derniers rapports sexuels à hauts risques [4].Le VIH et le SIDA sont des problèmes majeurs de santé publique dans le monde faisant de la jeunesse y compris le monde scolaire un groupe vulnérable du fait qu'elle n'est pas suffisamment bien préparée pour affronter les problèmes liés à leur sexualité et aussi du fait qu'elle ne dispose pas suffisamment de connaissances réelles sur l'infection à VIH et le SIDA [5].L'absence d'éducation sexuelle à l'école et à la maison est un facteur majeur, comme en témoigne le fait que $33 \%$ de toutes les grossesses et $26 \%$ de tous les avortements sont observés chez les moins de 20 ans qui constituent la population cible [6].Les Organisations Non Gouvernementales (ONG) intervenant dans la zone de Niono, dont certaines plus d'une décennie et d'autres récemment, ont mené des actions de sensibilisations en faveur de la promotion de la santé de la reproduction et de la prévention des Infections Sexuellement Transmissibles (IST-SIDA) à l'endroit de certaines couches y compris les élèves. Malgré ces efforts les IST, le VIH et le SIDA demeurent toujours un problème pour les adolescentes. Il est apparu important de procéder à une évaluation après les différentes séances d'information et de sensibilisation de la part des structures publiques de santé et des ONG afin de comprendre les facteurs dont la maîtrise pourrait contribuer à lutter efficacement contre la propagation des IST, du VIH et du SIDA en milieu scolaire à Niono. L'objectif était d'étudier les connaissances, attitudes et pratiques des jeunes lycéens de Niono au Mali en matière d'IST de VIH et du SIDA.

\section{MATERIEL ET METHODES}

Cadre d'étude : L'étude a eu lieu à Niono au lycée MICHEL ALLAIRE situé près de l'auto-gare au quartier B1 et celui public de Niono situé à KOULAMBA WERE à 7 Kilomètres de Niono.

Type et période d'étude : Il s'agissait d'une étude transversale descriptive qui s'est déroulée d'avril 2016 à mars 2017 soit une période de douze (12) mois.

Population d'étude : Elle était constituée des élèves âgés de 15 à 24 ans dans les deux lycées de Niono.

Critères d'inclusion : il s'est agi d'être lycéen dans un des établissements sélectionnés, âgé de 15 - 24 ans, consentir de participer librement et volontairement à l'étude et être présent dans l'établissement le jour de l'enquête. N'ont pas été inclus, ceux qui étaient dans l'incapacité de répondre aux questions.

La taille de l'échantillon : $\mathrm{La}$ taille de l'échantillon a été calculée suivant la formule de DANIEL SCHWARTZ, $n=\left(Z \alpha^{2} * \mathrm{p} * \mathrm{q}\right) / \mathrm{i}^{2} ;$ avec i (la précision souhaitée) $=5 \%, Z \alpha$ (écart réduit pour un $\alpha$ de 0,05$)=1,96, \quad p=40 \%$ : c'est la proportion d'élèves ayant une connaissance sur les IST (La plupart des études réalisées en milieu rural ont montré une proportion d'élèves ayant une connaissance sur les IST estimée à $40 \%$ (10)), q = $1-\mathrm{p}=60 \%$. $n=369$. 
Base de sondage : Elle était constituée par la liste des élèves de 15 à 24 ans inscrits par établissement pour l'année académique 2016 -2017.

Méthode d'échantillonnage : Pour le choix des établissements, nous avons procédé au choix aléatoire de deux lycées sur les 5 que comptait la ville de Niono. Pour cela nous avons utilisé la fonction alea d'Excel.

Nous avons ensuite effectué un échantillonnage aléatoire systématique de 369 élèves sur l'effectif total des élèves de 15 à 24 ans dans les 2 établissements, puis procédé à un échantillonnage proportionnel à la taille de chaque établissement. Il était de 64\% au Lycée Michel Allaire et 36\% au Lycée Publique. Nous avons fait le cumul des élèves des deux établissements et déterminé la part de chacun. Cette proportion a été appliquée à la taille de l'échantillon, afin de savoir la part d'échantillon à sélectionner dans chacun des établissements. Nous avons déterminé un pas de sondage par établissement et procédé au choix des participants à enquêter.

Technique de collecte des données : Les données ont été collectées grâce à un questionnaire individuel administré sous forme d'interview confidentielle en mode face à face.

Saisie et analyse et présentation des données : Les données ont été saisies dans EXCEL 2013, analysées avec SPSS 20.0. Elles ont été présentées sous formes de tableaux et de figures. Nous avons utilisé les fréquences pour les variables qualitatives et les moyennes et l'écart type pour les variables quantitatives. Nous avons fait une analyse bi variée avec le test de Khi2 de Pearson au seuil de significativité de 0,05 .

\section{Considérations éthiques :}

Nous avons informé et obtenu les autorisations des administrations scolaires dont le Centre d'Animation Pédagogique (CAP) et les Proviseurs des lycées. Nous avons donné d'amples informations aux élèves sur les objectifs et la garantie de la confidentialité de l'entretien et des données récoltées. Les participants choisis ont été informés et conviés à l'interview de façon confidentielle. Les noms et prénoms n'ont pas été recueillis. L'entretien s'est déroulé en un endroit discret loin des gens. Nous avons recueilli le consentement verbal de chaque participant.

\section{RESULTATS}

\section{Caractéristiques sociodémographiques}

Tous les 369 élèves prévus ont été enquêtés. L'âge le plus représenté dans notre étude était de 17 ans avec $25,2 \%$. L'âge moyen était de $17,6 \pm 1,8$ ans. Les hommes représentaient $56,1 \%$ avec un sex ratio de 1,28 (Tableau I).
Tableau I : sociodémographiques des jeunes lycéens

\begin{tabular}{lcc}
\hline Caractéristiques & N & \% \\
\hline Age & & \\
15 & 34 & 09,2 \\
16 & 67 & 18,2 \\
17 & 93 & 25,2 \\
18 & 79 & 21,4 \\
19 & 51 & 13,8 \\
20 & 21 & 5,7 \\
21 & 09 & 2,4 \\
22 & 08 & 2,2 \\
23 & 04 & 1,1 \\
24 & 03 & 0,8 \\
Sexe & & \\
$\quad$ M & 207 & 56,1 \\
F & 162 & 43,9 \\
Statut matrimonial & & \\
Célibataire & 347 & 94,0 \\
$\quad$ Marié & 22 & 6,0 \\
Mode de résidence & & \\
$\quad$ Avec un parent & 48 & 13,0 \\
Avec deux & 303 & 82,1 \\
parents & & 4,9 \\
Autres & 18 &
\end{tabular}

\section{Connaissance sur les IST, VIH et SIDA}

La quasi-totalité des élèves enquêtés avait entendu parler de maladie que l'on peut attraper par rapport sexuel soit $92,4 \%$. Notons que $95,9 \%$ des enquêtés, avaient entendu parler du VIH-SIDA et 89,2\% croyaient à son existence. La causerie était la source d'information la plus citée avec $43,9 \%$. Près de la moitié des élèves (42\%) ne connaissait pas les signes des IST. Le signe le plus cité était la leucorrhée avec $8,1 \%$. La connaissance de la définition de la séropositivité était mauvaise pour $28,5 \%$. Plus du tiers des enquêtés, soit $37,7 \%$, savaient qu'on peut attraper le VIH sans manifester les symptômes. (Tableau II). 
Tableau II : Connaissances générales des élèves sur les IST/VIH/SIDA

\begin{tabular}{|c|c|c|}
\hline Caractéristiques & $\mathbf{n}$ & $\%$ \\
\hline \multicolumn{3}{|c|}{$\begin{array}{c}\text { Entendu parler d'une maladie que l'on peut } \\
\text { attraper par rapport sexuel }\end{array}$} \\
\hline Oui & 341 & 92,4 \\
\hline \multicolumn{3}{|l|}{ Entendu parler de VIH-sida } \\
\hline Oui & 354 & 95,9 \\
\hline \multicolumn{3}{|c|}{ Croyance à l'existence du VIH-sida } \\
\hline Oui & 329 & 89,2 \\
\hline \multicolumn{3}{|l|}{ Source d'information } \\
\hline Causerie & 162 & 43,9 \\
\hline Radio & 53 & 14,4 \\
\hline Télévision & 104 & 28,2 \\
\hline Théâtre & 5 & 1,4 \\
\hline Ecole & 18 & 4,9 \\
\hline Parents & 3 &, 8 \\
\hline Agent de Santé & 6 & 1,6 \\
\hline Autres & 18 & 4,9 \\
\hline \multicolumn{3}{|c|}{ Connaissance d'exemples d'IST } \\
\hline Sida & 231 & 62,6 \\
\hline Syphilis & 16 & 4,3 \\
\hline Gonococcie & 86 & 23,3 \\
\hline Chancre mou & 5 & 1,4 \\
\hline Autres & 31 & 8,4 \\
\hline \multicolumn{3}{|c|}{ Connaissance des signes d'IST } \\
\hline Leucorrhée & 30 & 8,1 \\
\hline $\begin{array}{l}\text { Prurit }+ \text { Douleur } \\
\text { abdominale }\end{array}$ & 28 & 7,6 \\
\hline $\begin{array}{l}\text { Céphalée + fièvre + } \\
\text { vomissement }\end{array}$ & 137 & 37,1 \\
\hline Amaigrissement & 19 & 5,1 \\
\hline Ne Sais Pas & 155 & 42,0 \\
\hline \multicolumn{3}{|c|}{$\begin{array}{l}\text { Connaissance du lien entre VIH et ses } \\
\text { symptômes }\end{array}$} \\
\hline Oui & 139 & 37,7 \\
\hline
\end{tabular}

Le rapport sexuel était la voie de contamination la plus citée soit $32,2 \%$. L'utilisation du préservatif était le moyen de réduction de risque le plus cité avec 44,2\%. Les raisons de l'utilisation du préservatif étaient pour éviter la maladie et les grossesses avec respectivement $51,5 \%$ et $35,2 \%$, mais $13,3 \%$ affirmaient ne pas savoir. Plus de deux tiers des enquêtés (74\%) affirmaient que le risque des IST augmentait avec la multiplicité des partenaires. La plupart des enquêtés $(61,8 \%)$ pensaient qu'ils sont à l'abri d'IST. Près de la moitié de nos enquêtés $(47,4 \%)$ disait qu'il était possible de trouver le préservatif à la pharmacie. Plus d'un tiers de nos jeunes $(39,6 \%)$ pensaient que les routiers étaient le groupe le plus exposé.
Tableau III : Connaissance des élèves sur la transmission des IST/VIH/SIDA

\begin{tabular}{|c|c|c|}
\hline Caractéristiques & $\mathbf{n}$ & $\%$ \\
\hline \multicolumn{3}{|l|}{ Voies de transmission } \\
\hline Rapport sexuel & 119 & 32,2 \\
\hline Mère - enfant & 102 & 27,6 \\
\hline Sang et objets souillés & 127 & 34,4 \\
\hline Ne sais pas & 21 & 5,7 \\
\hline $\begin{array}{l}\text { Autres (piqure de moustiques, } \\
\text { embrassades) }\end{array}$ & 31 & 8,4 \\
\hline \multicolumn{3}{|l|}{ Mode de prévention } \\
\hline Utilisation du préservatif & 163 & 44,2 \\
\hline Abstinence du rapport sexuel & 133 & 36,0 \\
\hline $\begin{array}{l}\text { choix d'un partenaire non } \\
\text { infecte }\end{array}$ & 55 & 14,9 \\
\hline $\begin{array}{l}\text { Limitation du nombre de } \\
\text { partenaires }\end{array}$ & 18 & 4,9 \\
\hline
\end{tabular}

Augmentation du risque avec plusieurs partenaires

$\begin{array}{ccc}\text { Oui } & \mathbf{2 7 3} & \mathbf{7 4 , 0} \\ \text { Non } & 35 & 9,5 \\ \text { NSP } & 61 & 16,5\end{array}$

Lieux d'approvisionnement du préservatif

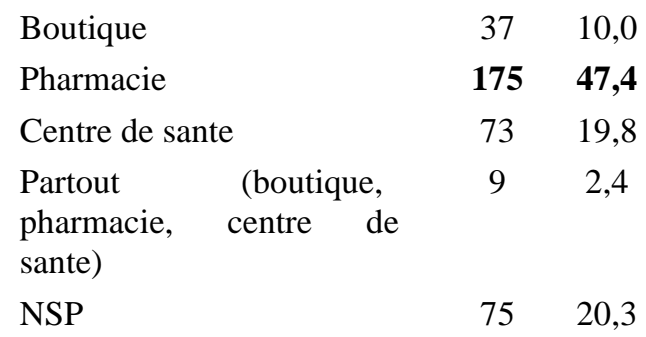

Groupe de population les plus exposés

\begin{tabular}{lcc} 
Routier & $\mathbf{1 4 6}$ & $\mathbf{3 9 , 6}$ \\
Migrant & 143 & 38,8 \\
Elèves & 29 & 7,9 \\
Professionnelles de sexe & 18 & 4,9 \\
Hétéro-homosexuels & 9 & 2,4 \\
Enseignant & 5 & 1,4 \\
Autres & 19 & 5,1 \\
\hline
\end{tabular}

Une proportion de $42 \%$ d'élèves a affirmé avoir déjà parlé de sexualité avec un de leurs parents. Plus de la moitié des jeunes lycéens (54,5\%) trouvait que la sexualité ne doit pas rester tabou entre parents et enfants. La majorité de nos enquêtés $(78 \%)$ étaient d'avis pour l'enseignement de la sexualité à l'école. Le second cycle était le niveau d'étude le mieux adapté pour l'enseignement de la sexualité pour plus d'un tiers $(39,6 \%)$ de nos enquêtés, suivi du Lycée $(37,7 \%)$ et du premier cycle $(22,8 \%)$.

Attitudes des jeunes lycéens face au dépistage des IST, VIH et SIDA 
Près de deux tiers des enquêtés ont affirmé l'acceptation volontaire du test de dépistage soit $62,9 \%$. Environ $25,5 \%$ ont affirmé le contraire et $11,7 \%$ ne savaient pas. Seulement le quart des enquêtés $(25,2 \%)$ a affirmé avoir déjà fait un test de dépistage volontaire. Sur les 232 élèves qui avaient opté pour le dépistage seulement 75 avaient affirmé l'avoir déjà fait. Nous avons trouvé une relation statistiquement significative entre l'option pour le dépistage et l'affirmation de la réalisation du dépistage déjà fait, $\mathrm{p}<10^{-5}$ (Tableau IV). La plupart des enquêtés $(71,50 \%)$ pensaient que les PV-VIH doivent être isolées.

Tableau IV : Répartition des jeunes lycéens qui avaient opté pour le dépistage et ceux qui l'avaient déjà fait

\begin{tabular}{|c|c|c|c|c|c|}
\hline & & \multicolumn{3}{|c|}{$\begin{array}{c}\text { Déjà fait un test de dé } \\
\text { pistage }\end{array}$} & \multirow[b]{2}{*}{ Total } \\
\hline & & Oui & Non & NSP & \\
\hline 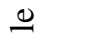 & Oui & 75 & 152 & 5 & 232 \\
\hline & Non & 13 & 77 & 4 & 94 \\
\hline 总 & NSP & 5 & 12 & 26 & 43 \\
\hline ô. & Total & 93 & 241 & 35 & 369 \\
\hline
\end{tabular}

\section{Pratiques et comportements sexuels des jeunes} en matière d'IST, VIH et SIDA

Près de la moitié, soit $47,2 \%$ des enquêtés, avait un partenaire sexuel au moment de l'enquête. Plus du tiers des enquêtés avaient affirmé avoir eu un contact sexuel, soit $35,2 \%$. Il y avait une relation statistiquement significative entre le sexe et le contact sexuel, $\mathrm{p}<0,005$. Les garçons avaient 1,5 fois plus de risque de contact sexuel que les filles. Parmi les 130 élèves qui avaient eu un contact sexuel, 18,5\% l'avaient eu entre 12-14ans, 70\% entre 15-18ans. Plus de deux tiers d'entre eux $(\mathrm{N}=130)$ soit $68,5 \%$ avaient eu leur premier contact par curiosité $(23,1 \%, \quad \mathrm{~N}=130)$ et par méconnaissance. Plus de la moitié d'entre eux $(54,6 \%, \mathrm{~N}=130)$ avaient utilisé le préservatif lors de leur $1^{\text {er }}$ contact sexuel. Sur l'ensemble de nos enquêtés ( $\mathrm{N}=369)$, seulement $43,6 \%$ avaient discuté de sexualité avec leurs partenaires.

Plus du tiers de nos enquêtés, soit $35 \%$, avait eu plus de trois partenaires au cours des trois dernières années précédant l'enquête. Cette fréquence était de $10,4 \%$ au cours des 12 derniers mois précédant l'enquête. Environ 11,7\% et 10,8\% avaient eu 2 à 3 partenaires respectivement au cours des trois dernières années précédant l'enquête et des 12 derniers mois précédant l'enquête. Les $16,5 \%$ et $23,8 \%$ avaient un partenaire et les $32,8 \%$ et $30,1 \%$ n'avaient aucun partenaire. Plus de la moitié des élèves en activité sexuelle (70/130 soit 53,8\%) avaient eu des rapports sexuels avec des partenaires occasionnels. Parmi eux 72,9\% (soit 51/70) ont affirmé avoir utilisé le préservatif. Plus de la moitié des enquêtés, soit $56,6 \%$, avaient affirmé qu'ils utiliseront le préservatif même si le partenaire sexuel s'y oppose.

\section{DISCUSSION}

Caractéristiques de la population d'étude

La majorité des enquêtés était de sexe masculin $(56 \%)$ avec un âge moyen de 17,62 ans, résidait pour la plupart avec leurs parents (82\%). Une étude similaire réalisée à Sikasso trouvait une prédominance féminine $(60,2 \%)$ avec une moyenne d'âge de 19,4 ans [7].

\section{Connaissances sur les IST, VIH et SIDA}

La plupart de nos enquêtés ont entendu parler de maladie qu'on peut attraper lors des rapports sexuels (92\%), des IST $(95,9 \%)$ et du VIH/SIDA $(89,2 \%)$. Les principales IST citées par nos enquêtés étaient : le VIH/Sida $(62,6 \%)$ suivi de la gonococcie $(23,3 \%)$. Thanavanh et al. trouvent que 97,7\% des étudiants étaient conscients que le VIH peut être transmis par des rapports sexuels [8] et une étude réalisée au Togo par Kokou et al. affirment que $92 \%$ des élèves connaissaient d'autres IST en dehors du VIH/SIDA [9]. Les IST les plus citées dans la revue documentaire sont : le SIDA, la gonococcie et la syphilis. La majorité des élèves (44\%) ne connaissaient pas les signes des IST. La leucorrhée était le signe le plus évoquée. Ceci pourrait s'expliquer par le fait que nos enquêtés fréquentaient moins les centre de santé. Ces résultats sont différents de ceux rapportés par Konde. [10] qui trouve 52,3\%. Les faibles fréquences concernant les voies de transmission au cours de cette étude, pourraient s'expliquer par le fait que les jeunes à Niono ont moins accès aux informations données par les médias malgré la présence d'ONG de lutte contre les IST, le VIH et le SIDA dans le Cercle y compris dans les deux lycées. Ces résultats sont différents de ceux trouvés par Paraniala et al. avec $91 \%$ pour la transmission par voie hétérosexuelle [11] et selon Sangho et al. les principales voies de transmission citées par les élèves étaient les rapports sexuels $(96,7 \%)$, la transfusion sanguine $(91,8 \%)$ [12]. Par contre une étude similaire faite au Brésil montre que $24 \%$ croient que l'on peut attraper le VIH/ Sida par la salive [13]. Dans l'étude Touré et al. [14] menée à Abidjan les voies de transmission les plus citées étaient : la transmission materno-fœtale $(99,9 \%)$, la transmission sexuelle $(89,2 \%)$, la transmission sanguine $(68,7 \%)$. Ces fréquences sont supérieures aux nôtres. Malgré les messages, les sensibilisations dans lesdits lycées, le travail des ONG en ville, en ce qui concerne l'utilisation du préservatif, il a été cité comme moyen de prévention par moins de la moitié de nos enquêtés. 
$\mathrm{Ci}$ chiffre est largement inférieur à celui trouvé dans une étude réalisé au Congo où $87,8 \%$ des élèves ont cité le préservation comme moyen de prévention [15]. Contrairement au résultats obtenus par notre étude, les principales sources d'information des élèves sur le VIH/SIDA était : l'école avec $54 \%$, les médias $44,2 \%$ et les centres de santé 10,2\% selon l'étude du Congo [15]. Malgré le fait que plus de la moitié $(60 \%)$ de nos enquêtés résidaient avec leurs parents, seulement $0,8 \%$ avait reçu des informations d'eux. Cela serait dû au fait que, non seulement, la causerie avec les parents n'est pas culturelle chez nous, mais aussi parler de sexe avec les enfants continue d'être un tabou. Ce fait est confirmé par cette étude montrant que la sexualité est un sujet tabou pour $66 \%$ des élèves [16]. Nos résultats en faveur de la causerie comme source d'information s'expliqueraient par le fait que des ONG intervenant dans la zone organisent dans ces écoles des causeries pour sensibiliser les élèves dans la lutte contre la pandémie du siècle. Plus de la moitié $(52,3 \%)$ de nos jeunes lycéens n'avaient pas eu de conversation sur la sexualité avec un de leurs parents. Ils avaient entendu parler de sexualité pour la première fois au second cycle (52,3\%). Par contre, 42,8\% ont eu un entretien sur la sexualité avec un des parents. Ce résultat est proche de celui de TRAORE A. [17] qui dans son étude révèle que $40 \%$ des élèves ont eu un entretien sur la sexualité avec leurs parents. L'éducation sexuelle faite par les parents doit être encouragée, car nombreux sont les adolescents qui se plaignaient de n'avoir jamais eu l'occasion de discuter de sexualité avec l'un de leurs parents.

\section{Attitudes des jeunes lycéens face au dépistage des IST, VIH et SIDA}

Dans notre étude, les lycéens ont affirmé accepter volontairement de faire le test de dépistage $(62,9 \%)$ et une proportion de $25,2 \%$ avaient déjà fait le dépistage. Notre résultat est similaire à celui de l'étude de l'étude similaire faite à Sikasso où seulement $39,1 \%$ des élèves ont déclaré avoir fait le test de dépistage [7]. Nos enquêtés ont une opinion contrastée à propos des PV-VIH. Ils ont affirmé pouvoir manger de la nourriture préparée par un PV-VIH dans $62,6 \%$ de cas. Cependant, la majorité de nos jeunes enquêtés pensaient qu'on doit les isoler soit $71,5 \%$. En comparant ces résultats, on note que le sida au lieu d'être considéré comme la maladie d'autrui suscite la peur chez les adolescents.

\section{Pratiques et comportements sexuels des jeunes en matière d'IST, VIH et SIDA}

Dans notre étude, près de la moitié des élèves enquêtes $(48,8 \%)$ n'avaient pas encore eu un contact sexuel. C'était une question sensible à laquelle certains (16\%) n'avaient pas voulu répondre. La proportion de ceux qui avaient déjà eu une première expérience sexuelle était de 35,2\%.
L'âge moyen au premier rapport sexuel était de 17,8 ans et les âges extrêmes étaient de 12 et 18 ans. L'âge du premier rapport sexuel est relativement précoce. Dans l'étude réalisée par Cissoko et al., 48\% avaient déjà eu des relations sexuelles [3] pendant que 54\%, l'avait déjà eu selon l'étude de Sikasso [7]. Plus de la moitié des adolescents sexuellement actifs (soit 17,6\% de l'échantillon) avait eu globalement plus de trois partenaires sexuels, soit 35\% d'entre eux. L'étude de Sikasso notifiait que $22,9 \%$ avait deux partenaires au cours des 12 derniers mois [7]. Dans l'étude de Touré et al. [14] faite à Abidjan, plus de la moitié des élèves enquêtes $(56,1 \%)$ a déjà eu des rapports sexuels. L'âge moyen au premier rapport sexuel était de 15,02 ans chez les filles et 15,28 ans chez les garçons. Tous les enquêtés en activité sexuelle ont eu au moins deux partenaires sexuels et $26,6 \%$ ont eu plus de 5 partenaires sexuels. Nous remarquons que nos élèves ont une activité sexuelle précoce dont les causes peuvent être le manque d'éducation sexuelle, d'une part au sein des familles et d'autres parts à l'école ou les cours ne traitent que de la biologie et de l'anatomie, et ne parlent pas de sexualité en tant que tel. D'autres raisons pourraient intervenir notamment : les réseaux sociaux, internet où les sites pornographiques sont gratuits et d'accès facile. Le contexte du premier rapport sexuel est un indicateur clé du risque potentiel de contraction d'une IST et marque de façon psychologique les jeunes. Ainsi nous avons demandé aux jeunes le caractère volontaire ou non volontaire de leur premier rapport sexuel. Ainsi les résultats donnent que $68,5 \%$ ont vécu leur premier rapport sexuel de façon libre et volontaire (par curiosité). Par contre 23,1\% l'ont fait de façon non consensuelle (par contrainte). Parmi les 130 adolescents en activité sexuelle, 59 (soit $45,4 \%$ ) ont affirmé avoir utilisé le préservatif au cours du premier rapport sexuel. En somme, $44,2 \%$ le connaissaient déjà comme méthode préventive contre les IST. Au total, $65,7 \%$ de ceux qui ont eu des rapports avec des partenaires occasionnels déclarent avoir utilisé le préservatif au cours de ses rapports. Ce résultat est similaire à ceux rapportés par Essomba et al. [18] qui montre que $37 \%$ des enquêtés disaient porter un préservatif à chaque rapport sexuel douteux, 27,9\% rapportaient ne pas en avoir utilisé. Par ailleurs, ils diffèrent de ceux Bowassa et al. [15] où préservatif était utilisé au cours des rapports sexuels par $83,9 \%$ élèves et Touré et al. [14] avec de $80 \%$. En effet bien que près de la moitié des adolescents enquêtés $(45,4 \%)$ aient cité le préservatif, comme moyen de prévention, son utilisation reste irrégulière au motif qu'il diminue le plaisir au cours de l'acte sexuel ou qu'il est refusé par le partenaire sexuel.

\section{CONCLUSION}

Cette étude nous montre que la bonne connaissance des élèves sur l'existence des IST, du VIH et du 
sida, est en déphasage avec leur connaissance des moyens de prévention même si plus de moitié ont affirmé qu'ils utiliseront le préservatif malgré l'opposition du partenaire. Nous supposons que cela soit le reflet du fait que plus de la moitié n'ont pas eu de conversation sur la sexualité avec leurs parents. En vue d'améliorer les connaissances, attitudes et pratiques de ces jeunes, un programme de formation réglementé et adapté aux réalités socioculturelles doit être mis en place par les responsables de l'éducation nationale en plus des séances de sensibilisation adaptée à leurs besoins.

$\mathrm{Au}$ regard de ces résultats une étude d'envergure nationale pourrait être menée afin de connaitre la situation du pays dans le but de planifier des actions de riposte.

\section{Conflict d'intérêt : Aucun}

\section{REFERENCES}

1. Fiche d'information 2019 - Dernières statistiques sur l'état de l'épidémie de sida | ONUSIDA [Internet]. [cité 2 févr 2020]. Disponible sur: https://www.unaids.org/fr/resources/fact-sheet

2. WORD BANK The World Bank'scommitment to HIV/AIDS in Africa: our agenda for Action, 2007-2011, Washington Mach 2008, 118p.

3. Cissoko Y, Traoré A, Sidibé LN, Maiga YI, Coulibaly M, Maiga Z, et al. Knowledge attitude and practice toward STI, HIV/AIDS among high school students in Gao (Northen-Mali in West Africa). Connaissances, attitudes et pratiques face aux IST VIH-SIDA chez les lycéens de Gao (NordMali Afrique de l'Ouest). Revue Malienne d'Infectiologie et de Microbiologie. 2014;4:12.

4. Cellule de Planification et de Statistique (CPS/SSDSPF), Institut National de la Statistique (INSTAT/MPATP),INFO-STAT et ICF International, 2014. Enquête Démographique et de Santé au Mali 2012-2013. Rockville,Maryland, USA : CPS, INSTAT, INFO-STAT et ICF International.547p.

5. Guindo OM, Liu A. Knowledge, Attitudes and Practices Regarding HIV/AIDS among undergraduate students in China and Mali. Revue Malienne d'Infectiologie et de Microbiologie. 2014;3:14.

6. OMS : organisation mondiale de la santé SIDA, prévention et lutte Genève 1988, 190p.

7. Kounta CH, Diarra S, Toumanion B, Doumbia L et Giani S. Etude des connaissances, des attitudes et des pratiques des jeunes lyceens en matière des IST $\mathrm{du}$ VIH et du SIDA dans la Commune urbaine de Sikasso, Mali. Revue Malienne d'Infectiologie et de Microbiologie. 2014;(1):7-10. [cité 29 juin 2020]; Disponible sur: http://revues.ml/index.php/remim/article/view/378

8. Thanavanh B, Harun-Or-Rashid M, Kasuya H, Sakamoto J. Knowledge, attitudes and practices regarding HIV/AIDS among male high school students in Lao People's Democratic Republic. Journal of the International AIDS society. 2013;16(1):17387.

9. Segbedji Kar, Djadou Ke, Tchagbele O-B, Azoumah Kd, Geraldo A, Agbeko F, Et Al. Sante Sexuelle des Adolescents Collegiens: Connaissances, Attitudes, et Pratiques Face aux Risques des Infections Sexuellement Transmissibles à Lome. Revue Africaine et Malgache de Recherche Scientifique/Sciences de la Santé. 2016;4(2):85-9.

10. Konde A. Etude sur les connaissances attitudes et pratiques sur les IST et VIH /SIDA au Lycée Notre Dame du Niger. Thèse Méd, Bamako 200990 p.

11. Lui PS, Sarangapany J, Begley K, Coote K, Kishore K. Medical and nursing students perceived knowledge, attitudes, and practices concerning human immunodeficiency virus. International Scholarly Research Notices. 2014:1-9.

12. Sangho H, Keïta AS, Kondé A, Coulibaly C, Keita HD, Guindo MT, et al. Connaissances, Attitudes et Pratiques sur Les IST et VIH/Sida chez les Lyceennes à Bamako. Mali Médical. 2012;27(3):123.

13. Fontes MB, Crivelaro RC, Scartezini AM, Lima DD, Garcia A de A, Fujioka RT. Facteurs déterminants des connaissances, des attitudes et des pratiques en MST/SIDA et hépatites virales parmis les jeunes âgés de 18 à 29 ans au Brésil. Ciência \& Saúde Coletiva. 2017;22:1343-52.

14. Touré B, Koffi K, Kouassi-Gohou V, Kokoun E, Angbo-Effi O, Koffi NM, et al. Connaissances, attitudes et pratiques des collégiens et lycéens d'Abidjan face au VIH/sida. Med Trop. 2005;65:346-8.

15. Bowassa GE, Okoko AR, Moyen E, Motoula-Latou D, Oko APG, Kambourou J, et al. Connaissances, attitudes et pratiques des jeunes scolarisés face au VIH/SIDA à Brazzaville. Revue Africaine et Malgache de Recherche Scientifique/Sciences de la Santé. 2016;3(2):85-8.

16. Ndiaye P, Fall A, Tal-Dia A, Faye A, Diongue M. Connaissances, attitudes et pratiques relatives aux IST/VIH/sida: cas des hommes ayant des rapports sexuels avec d'autres hommes au Sénégal. Revue d'épidémiologie et de santé publique. 2011;59(5):305-11.

17. TRAORE A. Connaissances, attitudes comportementales des jeunes de moins de vingt ans face aux IST/SIDA Thèse Pharm Bamako 2006 Connaissances, attitudes comportementales des jeunes de moins de vingt ans face aux IST/SIDA. Thèse Pharm Bamako 2006, 92p.

18. Essomba EN, Bita Fouda A, Lehman L. Comportement a risque de transmission du HIV des élèves et étudiants de Douala, Cameroun. Mali Médical. 2013;28(4):16-21. 